\title{
On Diagonal Stability of Positive Systems with Switches and Delays
}

\author{
A. Yu. Aleksandrov ${ }^{*, * * a}$ and O. Mason ${ }^{* * * * * * *, b}$ \\ * St. Petersburg State University, St. Petersburg, Russia \\ ** ITMO University (National Research University of Information Technologies, Mechanics and Optics), \\ St. Petersburg, Russia \\ *** National University of Ireland, Maynooth, Ireland \\ **** Irish Software Research Centre Lero, Limerick, Ireland \\ e-mail: ${ }^{a} a . u . a l e k s a n d r o v @ s p b u . r u,{ }^{b}$ oliver.mason@mu.ie \\ Received December 1, 2017
}

\begin{abstract}
We consider linear positive systems with delay and switchings of operation modes. We establish conditions under which it is possible to construct a common Lyapunov-Krasovskii diagonal functional for the family of subsystems corresponding to the system with switchings in consideration. These conditions are formulated in terms of the feasibility of auxiliary systems of linear algebraic inequalities. In addition, we study the problem of the existence of a diagonal functional of a special form. We also show that our results can be used to analyze the stability of some classes of nonlinear positive systems with delay.
\end{abstract}

Keywords: switching systems, delays, diagonal stability, positive system, linear inequalities

DOI: $10.1134 /$ S0005117918120020

\section{INTRODUCTION}

Stability analysis of positive systems is an important problem of modern control theory [1-4]. Positive systems are those for which the non-negative orthant of the phase space is a positively invariant set. Such systems are used to simulate economic, biological, chemical, and a number of other processes $[1,5-7]$. In recent years, they have found extensive applications in network control problems (see, for example, $[4,8]$ ). In addition, positive systems arise as the result of constructing comparison systems for complex systems $[5,9,10]$.

Positive systems have a number of important and interesting properties that are effectively used in the analysis of stability and the synthesis of controls for these systems. One of these properties is diagonal stability. It is known $[1,7]$ that a positive linear stationary system is asymptotically stable if and only if there exists a Lyapunov function for this system in the form of a quadratic form with a diagonal matrix. The use of diagonal Lyapunov functions is convenient due to their simple structure. They are successfully used to study the stability of population dynamics models and neural networks, as well as in decentralized control problems for complex systems (see $[1,2,7,11]$ ). In many cases, they make it possible to prove the robustness of stability with respect to parametric perturbations and delays $[1,3,7,12]$.

One very interesting extension of the diagonal stability property is to pass from linear stationary systems to wider classes of positive systems, including systems with delay, with switchings, and nonlinear systems. A series of results in these directions have been obtained in $[1,3,7,12-15]$.

It should be noted that modern literature has no general definition of diagonal stability. For a given system, the Lyapunov diagonal function can be chosen in different forms [7]. Thus, diagonal 
stability is not a property of the system itself, but of the "system-Lyapunov function" pair (or the "system-Lyapunov-Krasovskii functional" pair).

In this work, we study the conditions for diagonal stability of linear positive systems with delay and switchings of operation modes. A switching system is a hybrid dynamic system consisting of a family of subsystems and a switching law that determines which of the subsystems is active at every moment of time. In a wide class of cases, the design of controlled systems requires to ensure their stability for any valid switching law $[16,17]$. The main approach to proving stability, uniform with respect to the switching law, is to find a common Lyapunov function for a set of subsystems corresponding to the considered hybrid system [16, 18]. However, the problem of the existence of the common Lyapunov function has not yet been fully solved even for the family of linear stationary subsystems [16].

In this work, we establish conditions under which we can construct a common LyapunovKrasovskii diagonal functional for a family of subsystems corresponding to a given positive system with switchings. These conditions are formulated in terms of the feasibility of auxiliary systems of linear algebraic inequalities. In addition, we study the problem of the existence of a diagonal functional of a special form. We also show that the obtained results can be used to analyze the stability of some classes of nonlinear positive systems with delay.

\section{PRELIMINARIES AND PROBLEM SETTING}

Consider a linear stationary system

$$
\dot{x}(t)=A x(t)
$$

where $x(t)=\left(x_{1}(t), \ldots, x_{n}(t)\right)^{\top}$ is the system state vector, $A$ is a constant matrix, $\top$ denotes transposition.

It is known $[5,6]$ that system (1) is positive if and only if all off-diagonal elements in the matrix $A$ are nonnegative. Such matrices are called Metzler.

Definition 1. We say that system (1) is diagonally stable if for this system there exists a Lyapunov function in the form of a quadratic form with a diagonal matrix that satisfies the requirements of the Lyapunov theorem on asymptotic stability.

Statement 1 [1]. Let $A$ be a Metzler matrix. Then the following conditions are equivalent:

(a) A is a Hurwitz matrix;

(b) there exists a vector $\theta>0$ such that

$$
A \theta<0
$$

(c) there exists a vector $d>0$ such that

$$
A^{\top} d<0
$$

(d) there exists a diagonal positive definite matrix $P=\operatorname{diag}\left\{p_{1}, \ldots, p_{n}\right\}$ such that the matrix $A^{\top} P+P A$ is negative definite.

Hereinafter, we understand vector inequalities to hold componentwise.

Remark 1. It is known $[1,6]$ that if positive vectors $\theta=\left(\theta_{1}, \ldots, \theta_{n}\right)^{\top}$ and $d=\left(d_{1}, \ldots, d_{n}\right)^{\top}$ satisfy inequalities (2) and (3) respectively, then in the condition (d) of Statement 1 one such diagonal matrix $P$ is obtained by setting

$$
p_{i}=d_{i} / \theta_{i}, \quad i=1, \ldots, n .
$$

AUtOMATION AND REMOTE CONTROL Vol. $79 \quad$ No. 122018 
Remark 2. Statement 1 implies that for the diagonal stability of the positive system (1) it is necessary and sufficient that the matrix $A$ is Hurwitz.

Next, we consider a linear positive system with switchings

$$
\dot{x}(t)=A_{\sigma} x(t)
$$

and the corresponding family of subsystems

$$
\dot{x}(t)=A_{s} x(t), \quad s=1, \ldots, N,
$$

where $A_{1}, \ldots, A_{N}$ are constant Metzler matrices, $\sigma=\sigma(t)$ is a piecewise constant function that defines the switching law, $\sigma(t):[0,+\infty) \mapsto\{1, \ldots, N\}$. For all hybrid systems considered in this work, we will assume that function $\sigma(t)$ on any bounded interval has a finite number of discontinuity points. Such switching laws will be called admissible.

Statement 2 [15]. Suppose that there exist numbers $\lambda, \mu$ and positive vectors $\theta=\left(\theta_{1}, \ldots, \theta_{n}\right)^{\top}$, $d=\left(d_{1}, \ldots, d_{n}\right)^{\top}$ such that

$$
\lambda+\mu<0, \quad A_{s} \theta \leqslant \lambda \theta, \quad A_{s}^{\top} d \leqslant \mu d, \quad s=1, \ldots, N .
$$

If $P=\operatorname{diag}\left\{d_{1} / \theta_{1}, \ldots, d_{n} / \theta_{n}\right\}$, then the matrices

$$
A_{s}^{\top} P+P A_{s}, \quad s=1, \ldots, N,
$$

are negative definite.

Thus, if conditions of Statement 2 are satisfied for family (5), there is a common Lyapunov diagonal function $V(x)=x^{\top} P x$.

Consider now a linear system with delay

$$
\dot{x}(t)=A x(t)+B x(t-\tau) .
$$

Here $x(t) \in \mathbb{R}^{n}, A$ and $B$ are constant matrices, $\tau$ is a constant non-negative delay. By $x_{t}$ we denote a segment of the solution of system (7): $x_{t}: \xi \mapsto x(t+\xi), \xi \in[-\tau, 0]$. It is known [5, 12] that system (7) is positive if and only if $A$ is Metzler and $B$ is a non-negative matrix.

Definition 2. We say that system (7) is diagonally stable if for this system one can construct a Lyapunov-Krasovskii type functional

$$
V\left(x_{t}\right)=x^{\top}(t) P x(t)+\int_{t-\tau}^{t} x^{\top}(u) Q x(u) d u
$$

with diagonal positive definite matrices $P$ and $Q$ that for all $\tau>0$ satisfy the requirements of the Krasovskii theorem on asymptotic stability [19].

Statement 3 [20]. Suppose that matrix $A$ is Metzler and $B$ is nonnegative. Then for the diagonal stability of system (7) it is necessary and sufficient that matrix $A+B$ is Hurwitz.

Remark 3. Results obtained in [20] imply that if conditions of Statement 3 are satisfied, then diagonal elements of matrix $P$ can be determined by formulas (4), where $d_{i}$ and $\theta_{i}$ are the components of positive vectors $\theta$ and $d$ satisfying inequalities $(A+B) \theta<0$ and $(A+B)^{\top} d<0$ respectively.

In this work, we consider a linear positive system with delay and switchings

$$
\dot{x}(t)=A_{\sigma} x(t)+B_{\sigma} x(t-\tau) .
$$

Here $x(t) \in \mathbb{R}^{n}, A_{s}$ are constant Metzler matrices, $B_{s}$ are constant non-negative matrices, $\sigma=\sigma(t)$ is an admissible switching law, $\sigma(t):[0,+\infty) \mapsto\{1, \ldots, N\}, \tau$ is a constant non-negative delay. 
Definition 3. We say that system (9) is diagonally stable if for the corresponding family of subsystems

$$
\dot{x}(t)=A_{s} x(t)+B_{s} x(t-\tau), \quad s=1, \ldots, N,
$$

there exists a common Lyapunov-Krasovskii functional (8) with diagonal positive definite matrices $P$ and $Q$ that satisfies for all $\tau>0$ the conditions of the Krasovskii theorem on asymptotic stability [19].

We study the conditions for the diagonal stability of system (9). In addition, we show that the resulting conditions can be used to analyze the stability of certain types of nonlinear systems with delay and switching operation modes.

\section{SUFFICIENT CONDITIONS FOR DIAGONAL STABILITY}

Statement 3 implies that system (7) with a Metzler matrix $A$ and a non-negative matrix $B$ is diagonally stable if and only if the corresponding delay-free system $\dot{x}(t)=(A+B) x(t)$ is diagonally stable. Based on this fact, we can suppose that if for a family of subsystems without delay

$$
\dot{x}(t)=\left(A_{s}+B_{s}\right) x(t), \quad s=1, \ldots, N,
$$

with Metzler matrices $A_{s}$ and non-negative matrices $B_{s}$ there exists a common Lyapunov function in the form of a quadratic form with a diagonal matrix, then for the corresponding family (10) there exists a common diagonal Lyapunov-Krasovskii functional of type (8). However, this hypothesis is incorrect.

Indeed, suppose that family (10) consists of two second-order subsystems $(n=2, N=2)$ with matrices

$$
A_{1}=\left(\begin{array}{rr}
-2 & 0 \\
0 & -3
\end{array}\right), \quad A_{2}=\left(\begin{array}{rr}
-4 & 0 \\
0 & -1
\end{array}\right), \quad B_{1}=B_{2}=\left(\begin{array}{ll}
0 & 2 \\
1 & 0
\end{array}\right) .
$$

In this case, function $V\left(x_{1}, x_{2}\right)=x_{1}^{2}+x_{2}^{2}$ is the general diagonal Lyapunov function for the corresponding family (11). At the same time, it is easy to show that family (10) has no diagonal Lyapunov-Krasovskii functional (8).

To obtain sufficient conditions for the diagonal stability of the system (9), we use the approaches proposed in $[15,21]$.

Theorem 1. Let $A_{s}$ be Metzler, and let $B_{s}$ be nonnegative matrices, $s=1, \ldots, N$. If there exist numbers $\lambda, \mu$ and positive vectors $\theta, d$ such that

$$
\begin{gathered}
\lambda+\mu<0, \quad\left(A_{s}+B_{s}\right) \theta \leqslant \lambda \theta, \quad s=1, \ldots, N, \\
\left(A_{s}+B_{r}\right)^{\top} d \leqslant \mu d, \quad s, r=1, \ldots, N,
\end{gathered}
$$

then system (9) is diagonally stable.

Proof of Theorem 1, as well as Theorems 3, 4, and Corollary 2 are given in the Appendix.

Remark 4. The proof of Theorem 1 contains a constructive algorithm for finding the required functional.

Corollary 1. Let $A_{s}$ be Metzler, and let $B_{s}$ be nonnegative matrices, $s=1, \ldots, N$. If there exist positive vectors $\theta$, $d$ such that

$$
\begin{gathered}
\left(A_{s}+B_{s}\right) \theta<0, \quad s=1, \ldots, N, \\
\left(A_{s}+B_{r}\right)^{\top} d<0, \quad s, r=1, \ldots, N,
\end{gathered}
$$

then system (9) is diagonally stable.

AUTOMATION AND REMOTE CONTROL Vol. 79 No. 122018 
When analyzing the stability of linear systems with switchings, of particular interest are the cases when matrices of the subsystems commute [22] or generate a solvable Lie algebra [23]. It is known $[16,22]$ that if in the family (5) matrices $A_{1}, \ldots, A_{N}$ are Hurwitz (and they do not have to be Metzler) and satisfy the conditions $A_{s} A_{r}=A_{r} A_{s}, s, r=1, \ldots, N$, then there exists a constant positive matrix $P$ (not necessarily diagonal) for which matrices (6) are negative definite. It was shown in [13] that under the additional assumption that $A_{1}, \ldots, A_{N}$ are Metzler matrices the required matrix $P$ can be chosen to be diagonal. The case of commutative matrices is also interesting for the problem considered in this work.

Corollary 2. Let $A_{s}$ be Metzler and let $B_{s}$ be nonnegative matrices, $s=1, \ldots, N$. If matrices $A_{s}+B_{r}, s, r=1, \ldots, N$ are commutative and Hurwitz, then system (9) is diagonally stable.

Theorem 1 can be extended to systems with several delays.

Consider a hybrid system

$$
\dot{x}(t)=A_{\sigma} x(t)+B_{\sigma}^{(1)} x\left(t-\tau_{1}\right)+\ldots+B_{\sigma}^{(m)} x\left(t-\tau_{m}\right)
$$

and the corresponding family of subsystems

$$
\dot{x}(t)=A_{s} x(t)+B_{s}^{(1)} x\left(t-\tau_{1}\right)+\ldots+B_{s}^{(m)} x\left(t-\tau_{m}\right), \quad s=1, \ldots, N .
$$

Here $x(t) \in \mathbb{R}^{n}, A_{s}$ and $B_{s}^{(j)}$ are constant matrices, $s=1, \ldots, N, j=1, \ldots, m, \sigma=\sigma(t)$ is an admissible switching law, $\sigma(t):[0,+\infty) \mapsto\{1, \ldots, N\}, \tau_{1}, \ldots, \tau_{m}$ are constant non-negative delays.

Theorem 2. Let $A_{s}$ be Metzler and $B_{s}^{(j)}$ be non-negative matrices, $s=1, \ldots, N, j=1, \ldots, m$. If there exist numbers $\lambda, \mu$ and positive vectors $\theta, d$ such that $\lambda+\mu<0$,

$$
\begin{gathered}
\left(A_{s}+B_{s}^{(1)}+\ldots+B_{s}^{(m)}\right) \theta \leqslant \lambda \theta, \quad s=1, \ldots, N, \\
\left(A_{s}+B_{r_{1}}^{(1)}+\ldots+B_{r_{m}}^{(m)}\right)^{\top} d \leqslant \mu d, \quad s, r_{1}, \ldots, r_{m}=1, \ldots, N,
\end{gathered}
$$

then for the family (14) we can construct a common Lyapunov-Krasovskii functional

$$
V\left(x_{t}\right)=x^{\top}(t) P x(t)+\int_{t-\tau_{1}}^{t} x^{\top}(u) Q^{(1)} x(u) d u+\ldots+\int_{t-\tau_{m}}^{t} x^{\top}(u) Q^{(m)} x(u) d u
$$

with positive definite diagonal matrices $P, Q^{(1)}, \ldots, Q^{(m)}$.

Proof of this theorem is similar to the proof of Theorem 1.

\section{CONDITIONS FOR THE EXISTENCE OF A DIAGONAL FUNCTIONAL OF A SPECIAL TYPE}

The work [24] investigated diagonal stability of linear positive systems of the form (1). Based on the duality principle, the work established a criterion for the existence of a quadratic Lyapunov function $V(x)=x^{\top} P x$ with a diagonal matrix $P$, where a part of the diagonal elements are equal to one, i.e.,

$$
P=\left(\begin{array}{ll}
I & 0 \\
0 & \tilde{P}
\end{array}\right)
$$

Here $I$ is the identity matrix of dimension $l, \tilde{P}$ is a diagonal positive definite matrix of dimension $n-l, 1 \leqslant l<n$.

In this section, we study the conditions under which we can construct a Lyapunov-Krasovskii functional of a diagonal type (8) with identity matrix $P$ for a linear positive system with delay.

First, we consider the system without switching (7). 
Theorem 3. Let $A$ be Metzler, and let $B$ be a non-negative matrix. Then in order for system (7) to have a diagonal Lyapunov-Krasovskii functional (8) with the identity matrix $P$, it is necessary and sufficient that the matrix

$$
C=A+B+(A+B)^{\top}
$$

is negative definite.

We now consider the hybrid system (9).

Theorem 4. Let $A_{s}$ be Metzler and let $B_{s}$ be non-negative matrices, $s=1, \ldots, N$. If there exists a positive vector $\zeta$ such that

$$
\left(A_{s}+B_{s}+A_{s}^{\top}+B_{r}^{\top}\right) \zeta<0, \quad s, r=1, \ldots, N
$$

then for the family (10) one can construct a common Lyapunov-Krasovskii functional of the form (8) with identity matrix $P$.

\section{APPLICATIONS OF THE OBTAINED RESULTS TO THE ANALYSIS OF THE STABILITY OF NONLINEAR SYSTEMS}

\subsection{Systems with Separable Nonlinearities of Sector Type}

Suppose that we are given a nonlinear switching system

$$
\dot{x}(t)=A_{\sigma} f(x(t))+B_{\sigma} f(x(t-\tau))
$$

and the corresponding family of subsystems

$$
\dot{x}(t)=A_{s} f(x(t))+B_{s} f(x(t-\tau)), \quad s=1, \ldots, N .
$$

Here $x(t) \in \mathbb{R}^{n}, A_{s}$ and $B_{s}$ are constant matrices, $\sigma=\sigma(t)$ is an admissible switching law, the vector function $f(x)$ is defined and continuous for $\|x\|<H(0<H \leqslant+\infty,\|\cdot\|$ is the Euclidean norm of a vector), $\tau$ is a constant non-negative delay. We assume that $f(x)$ is a separable nonlinearity, i.e., $f(x)=\left(f_{1}\left(x_{1}\right), \ldots, f_{n}\left(x_{n}\right)\right)^{\top}$, and that scalar functions $f_{i}\left(x_{i}\right)$ satisfy the following sector constraints: $x_{i} f_{i}\left(x_{i}\right)>0$ with $x_{i} \neq 0, i=1, \ldots, n$. Such functions will be called admissible. Systems of the form (17) are widely used in modeling automatic control systems and neural networks (see $[1,5,7])$.

Consider the functional

$$
V\left(x_{t}\right)=2 \sum_{i=1}^{n} p_{i} \int_{0}^{x_{i}(t)} f_{i}(\eta) d \eta+\sum_{i=1}^{n} q_{i} \int_{t-\tau}^{t} f_{i}^{2}\left(x_{i}(u)\right) d u,
$$

where $p_{i}, q_{i}$ are constant positive coefficients.

We choose an index $s \in\{1, \ldots, N\}$ and differentiate the functional (19) with respect to the sth subsystem of family (18). We get

$$
\left.\dot{V}\right|_{(s)}=\left(\begin{array}{c}
f(x(t)) \\
f(x(t-\tau))
\end{array}\right)^{\top}\left(\begin{array}{cc}
A_{s}^{\top} P+P A_{s}+Q & P B_{s} \\
B_{s}^{\top} P & -Q
\end{array}\right)\left(\begin{array}{c}
f(x(t)) \\
f(x(t-\tau))
\end{array}\right) .
$$

Here $P=\operatorname{diag}\left\{p_{1}, \ldots, p_{n}\right\}, Q=\operatorname{diag}\left\{q_{1}, \ldots, q_{n}\right\}$.

Suppose that conditions of Theorem 1 are satisfied. Then positive numbers $p_{1}, \ldots, p_{n}, q_{1}, \ldots, q_{n}$ can be chosen in such a way that the following estimates hold:

$$
\left.\dot{V}\right|_{(s)} \leqslant-\beta\left(\|f(x(t))\|^{2}+\|f(x(t-\tau))\|^{2}\right), \quad s=1, \ldots, N, \quad \beta=\text { const }>0 .
$$

Hence, the zero solution of system (17) will be asymptotically stable for any admissible nonlinearities, any admissible switching law, and any non-negative delay. 


\subsection{Systems with Non-Conservative Perturbations}

Along with system (10), we consider a perturbed system

$$
\dot{x}(t)=A_{\sigma} x(t)+B_{\sigma} x(t-\tau)+G(t, x(t), x(t-\tau)) x(t),
$$

where $G(t, x, y)$ is a skew-symmetric matrix $\left(G^{\top}=-G\right)$ continuous for $t \geqslant 0, x, y \in \mathbb{R}^{n}$. Thus, the disturbing forces are non-conservative: $x^{\top} G(t, x, y) x=0$.

Suppose that matrices $A_{s}, B_{s}, s=1, \ldots, N$, possess the properties specified in Theorem 4 . Then for system (20) we can construct a diagonal functional of Lyapunov-Krasovskii type (8) with

identity matrix $P$. For the derivative of this functional with respect to the perturbed system we get the estimate

$$
\dot{V} \leqslant-\beta\left(\|x(t)\|^{2}+\|x(t-\tau)\|^{2}\right), \quad \beta=\text { const }>0 .
$$

Therefore, if the conditions of Theorem 4 are satisfied, then for any continuous skew-symmetric matrix $G$, any admissible switching law, and any non-negative delay, the zero solution of system (20) will be globally asymptotically stable.

\subsection{Construction of a "Potential" Functional}

Consider now the hybrid system

$$
\dot{x}(t)=A_{\sigma} \frac{\partial W(x(t))}{\partial x}+B_{\sigma} \frac{\partial W(x(t-\tau))}{\partial x}
$$

and the corresponding family of subsystems

$$
\dot{x}(t)=A_{s} \frac{\partial W(x(t))}{\partial x}+B_{s} \frac{\partial W(x(t-\tau))}{\partial x}, \quad s=1, \ldots, N .
$$

Here $x(t) \in \mathbb{R}^{n}, A_{s}$ and $B_{s}$ are constant matrices, $s=1, \ldots, N, \sigma=\sigma(t)$ is an admissible switching law, the function $W(x)$ is continuously differentiable for $\|x\|<H(0<H \leqslant+\infty)$ and positive definite, $\tau$ is a constant non-negative delay. In addition, suppose that $\partial W(x) / \partial x=0$ if and only if $x=0$. For example, this assumption is satisfied if $W(x)$ is a continuously differentiable positive definite homogeneous function of order $\nu>1$.

Remark 5. It is obvious that system (17) is a special case of system (21).

For family (22), we construct a common Lyapunov-Krasovskii functional in the form

$$
V\left(x_{t}\right)=W(x(t))+\int_{t-\tau}^{t}\left(\frac{\partial W(x(u))}{\partial x}\right)^{\top} Q \frac{\partial W(x(u))}{\partial x} d u
$$

where $Q$ is a constant positive definite diagonal matrix.

Fix an index $s \in\{1, \ldots, N\}$. Differentiating the functional (23) with respect to the $s$ th subsystem of family (22), we have

$$
\left.\dot{V}\right|_{(s)}=\left(\begin{array}{c}
\frac{\partial W(x(t))}{\partial x} \\
\frac{\partial W(x(t-\tau))}{\partial x}
\end{array}\right)^{\top}\left(\begin{array}{cc}
A_{s}^{\top}+A_{s}+Q & B_{s} \\
B_{s}^{\top} & -Q
\end{array}\right)\left(\begin{array}{c}
\frac{\partial W(x(t))}{\partial x} \\
\frac{\partial W(x(t-\tau))}{\partial x}
\end{array}\right) .
$$

AUTOMATION AND REMOTE CONTROL Vol. 79 No. 122018 
If the conditions of Theorem 4 are satisfied, then there exists a positive definite diagonal matrix $Q$ such that

$$
\begin{gathered}
\left.\dot{V}\right|_{(s)} \leqslant-\beta\left(\left\|\frac{\partial W(x(t))}{\partial x}\right\|^{2}+\left\|\frac{\partial W(x(t-\tau))}{\partial x}\right\|^{2}\right), \\
s=1, \ldots, N, \quad \beta=\text { const }>0 .
\end{gathered}
$$

Therefore, the zero solution of the system (21) will be asymptotically stable for any function $W(x)$ with the above properties, any admissible switching law, and any non-negative delay.

\subsection{A Discrete Switching System with Small Parameter}

Consider a discrete system

$$
x(k+1)=\left(R_{\sigma}+\mu D_{\sigma}\right) f(x(k))+\mu M_{\sigma} f(x(k-l)) .
$$

Here $x(k) \in \mathbb{R}^{n}$, the function $\sigma=\sigma(k)$ defines the switching law, $\sigma(k) \in\{1, \ldots, N\}, R_{s}, D_{s}, M_{s}$ are constant matrices, $s=1, \ldots, N, f(x)=\left(f_{1}\left(x_{1}\right), \ldots, f_{n}\left(x_{n}\right)\right)^{\top}$ is a separable nonlinearity, $l$ is a natural delay, $\mu$ is a small positive parameter, $k=0,1,2, \ldots$.

As admissible functions $f_{i}\left(x_{i}\right)$ we consider functions continuous for $\|x\|<H(0<H \leqslant+\infty)$ that satisfy the following conditions:

$$
x_{i} f_{i}\left(x_{i}\right)>0 \quad \text { for } x_{i} \neq 0, \quad\left|f_{i}\left(x_{i}\right)\right| \leqslant\left|x_{i}\right|, \quad i=1, \ldots, n \text {. }
$$

Switchings in system (24) are carried out between subsystems of the family

$$
x(k+1)=\left(R_{s}+\mu D_{s}\right) f(x(k))+\mu M_{s} f(x(k-l)), \quad s=1, \ldots, N .
$$

Systems of the form (25) are widely used as discrete models of digital filters and neural networks, as well as in the development of iterative numerical methods (see [7]).

Consider the case where $R_{1}, \ldots, R_{N}$ are orthogonal matrices $\left(R_{s}^{\top} R_{s}=I, s=1, \ldots, N\right)$. Then, for any switching law, the zero solution of the system

$$
x(k+1)=R_{\sigma} x(k),
$$

resulting from (24) with $\mu=0$ and $f(x)=x$, is not asymptotically stable.

For the family (25) we construct a common Lyapunov-Krasovskii functional in the form

$$
V\left(x^{(k)}\right)=x^{\top}(k) x(k)+\mu \sum_{j=1}^{l} f^{\top}(x(k-j))(Q+\mu(l-j+1) I) f(x(k-j)),
$$

where

$$
x^{(k)}=\left(x^{\top}(k), x^{\top}(k-1), \ldots, x^{\top}(k-l)\right)^{\top},
$$

and $Q$ is a constant positive definite diagonal matrix. 
Let us fix an index $s \in\{1, \ldots, N\}$ and calculate the increment of the functional (26) on solutions of the $s$ th subsystem of the family (25). We get

$$
\begin{gathered}
\left.\Delta V\right|_{(s)}=f^{\top}(x(k)) f(x(k))-x^{\top}(k) x(k)+\mu f^{\top}(x(k)) R_{s}^{\top}\left(D_{s} f(x(k))+M_{s} f(x(k-l))\right) \\
+\mu\left(D_{s} f(x(k))+M_{s} f(x(k-l))\right)^{\top} R_{s} f(x(k))+\mu^{2}\left\|D_{s} f(x(k))+M_{s} f(x(k-l))\right\|^{2} \\
+\mu f^{\top}(x(k)) Q f(x(k))-\mu f^{\top}(x(k-l)) Q f(x(k-l))+l \mu^{2}\|f(x(k))\|^{2}-\mu^{2} \sum_{j=1}^{l}\|f(x(k-j))\|^{2} \\
\leqslant \mu\left(\begin{array}{c}
f(x(k)) \\
f(x(k-l))
\end{array}\right)^{\top}\left(\begin{array}{cc}
A_{s}^{\top}+A_{s}+Q & B_{s} \\
B_{s}^{\top} & -Q
\end{array}\right)\left(\begin{array}{c}
f(x(k)) \\
f(x(k-l))
\end{array}\right) \\
+c \mu^{2}\left(\|f(x(k))\|^{2}+\|f(x(k-l))\|^{2}\right)-\mu^{2} \sum_{j=1}^{l}\|f(x(k-j))\|^{2} .
\end{gathered}
$$

Here $A_{s}=R_{s}^{\top} D_{s}, B_{s}=R_{s}^{\top} M_{s}, c$ is a positive constant.

If matrices $A_{s}, B_{s}, s=1, \ldots, N$, possess the properties specified in Theorem 4 , then there exist a number $\beta>0$ and a positive definite diagonal matrix $Q$ such that

$$
\left.\Delta V\right|_{(s)} \leqslant-\mu(\beta-c \mu)\left(\|f(x(k))\|^{2}+\|f(x(k-l))\|^{2}\right)-\mu^{2} \sum_{j=1}^{l}\|f(x(k-j))\|^{2}, \quad s=1, \ldots, N .
$$

Therefore, there exists $\mu_{0}>0$ such that for all $\mu \in\left(0, \mu_{0}\right)$ the zero solution of system (24) is asymptotically stable for any admissible functions $f_{1}\left(x_{1}\right), \ldots, f_{n}\left(x_{n}\right)$, any admissible switching law, and any positive integer delay.

\section{EXAMPLE}

Consider an automatic control system defined by the following equations with switchings and delay in feedback:

$$
\begin{aligned}
\dot{x}_{i}(t) & =a_{i}^{(\sigma)} x_{i}(t)+b_{i}^{(\sigma)} \varphi\left(x_{n}(t-\tau)\right), \quad i=1, \ldots, n-1, \\
\dot{x}_{n}(t) & =\sum_{j=1}^{n-1} c_{j}^{(\sigma)} x_{j}(t)+a_{n}^{(\sigma)} \varphi\left(x_{n}(t)\right),
\end{aligned}
$$

where $\sigma=\sigma(t)$ is an admissible switching law, $\sigma(t):[0,+\infty) \mapsto\{1, \ldots, N\} ; a_{j}^{(s)}, c_{i}^{(s)}, b_{i}^{(s)}$ are constant coefficients, and $a_{j}^{(s)}<0, c_{i}^{(s)}>0, b_{i}^{(s)}>0, i=1, \ldots, n-1, j=1, \ldots, n, s=1, \ldots, N$; $\varphi\left(x_{n}\right)$ is a scalar nonlinearity continuous for $\left|x_{n}\right|<H(0<H \leqslant+\infty)$ and satisfying a sector type condition $x_{n} \varphi\left(x_{n}\right)>0$ for $x_{n} \neq 0$; $\tau$ is a constant non-negative delay. Thus, at every moment of time one of the subsystems in the following family is active:

$$
\begin{aligned}
\dot{x}_{i}(t) & =a_{i}^{(s)} x_{i}(t)+b_{i}^{(s)} \varphi\left(x_{n}(t-\tau)\right), \quad i=1, \ldots, n-1, \\
\dot{x}_{n}(t) & =\sum_{j=1}^{n-1} c_{j}^{(s)} x_{j}(t)+a_{n}^{(s)} \varphi\left(x_{n}(t)\right), \quad s=1, \ldots, N .
\end{aligned}
$$

System (27) is a special case of system (17) (here $f_{i}\left(x_{i}\right)=x_{i}, i=1, \ldots, n-1, f_{n}\left(x_{n}\right)=\varphi\left(x_{n}\right)$ ). 
By considering the system matrices,

$$
A_{s}=\left(\begin{array}{ccccc}
a_{1}^{(s)} & 0 & \cdots & 0 & 0 \\
0 & a_{2}^{(s)} & \cdots & 0 & 0 \\
\vdots & \vdots & \ddots & \vdots & \vdots \\
0 & 0 & \cdots & a_{n-1}^{(s)} & 0 \\
c_{1}^{(s)} & c_{2}^{(s)} & \cdots & c_{n-1}^{(s)} & a_{n}^{(s)}
\end{array}\right), \quad B_{s}=\left(\begin{array}{ccccc}
0 & 0 & \cdots & 0 & b_{1}^{(s)} \\
0 & 0 & \cdots & 0 & b_{2}^{(s)} \\
\vdots & \vdots & \ddots & \vdots & \vdots \\
0 & 0 & \cdots & 0 & b_{n-1}^{(s)} \\
0 & 0 & \cdots & 0 & 0
\end{array}\right), \quad s=1, \ldots, N
$$

and applying the conditions of Theorem 1, we find that if there exist numbers $\lambda$ and $\mu$ such that

$$
\begin{gathered}
\lambda+\mu<0, \quad \lambda>\max _{j=1, \ldots, n} \max _{s=1, \ldots, N} a_{j}^{(s)}, \quad \mu>\max _{j=1, \ldots, n} \max _{s=1, \ldots, N} a_{j}^{(s)}, \\
\sum_{i=1}^{n-1} c_{i}^{(s)} \max _{l=1, \ldots, N} \frac{b_{i}^{(l)}}{\lambda-a_{i}^{(l)}} \leqslant \lambda-a_{n}^{(s)}, \quad s=1, \ldots, N, \\
\sum_{i=1}^{n-1} b_{i}^{(r)} \max _{l=1, \ldots, N} \frac{c_{i}^{(l)}}{\mu-a_{i}^{(l)}}+\max _{s=1, \ldots, N} a_{n}^{(s)} \leqslant \mu, \quad r=1, \ldots, N,
\end{gathered}
$$

then for the family (28) one can construct a common Lyapunov-Krasovskii functional of the form (19).

The conditions of Corollary 1 are satisfied for the matrices (29) if and only if

$$
\begin{gathered}
\sum_{i=1}^{n-1} c_{i}^{(s)} \max _{l=1, \ldots, N} \frac{b_{i}^{(l)}}{\left|a_{i}^{(l)}\right|}<\left|a_{n}^{(s)}\right|, \quad s=1, \ldots, N, \\
\sum_{i=1}^{n-1} b_{i}^{(r)} \max _{l=1, \ldots, N} \frac{c_{i}^{(l)}}{\left|a_{i}^{(l)}\right|}+\max _{s=1, \ldots, N} a_{n}^{(s)}<0, \quad r=1, \ldots, N .
\end{gathered}
$$

Applying Theorem 4, we get that if the following inequalities hold:

$$
\sum_{i=1}^{n-1}\left(c_{i}^{(s)}+b_{i}^{(r)}\right) \max _{l=1, \ldots, N} \frac{1}{\left|a_{i}^{(l)}\right|}\left(c_{i}^{(l)}+b_{i}^{(l)}\right)<4\left|a_{n}^{(s)}\right|, \quad s, r=1, \ldots, N,
$$

then for the family (28) there exists a common Lyapunov-Krasovskii type functional (19) with the identity matrix $P$.

Suppose that the family (28) consists of two third-order subsystems $(n=3, N=2)$ with the following coefficients:

$$
\begin{gathered}
a_{1}^{(1)}=a, \quad a_{1}^{(2)}=-4, \quad a_{2}^{(1)}=a_{2}^{(2)}=-6, \quad a_{3}^{(1)}=-4, \quad a_{3}^{(2)}=-8, \\
b_{1}^{(1)}=2, \quad b_{1}^{(2)}=1, \quad b_{2}^{(1)}=b_{2}^{(2)}=1, \\
c_{1}^{(1)}=1, \quad c_{1}^{(2)}=2, \quad c_{2}^{(1)}=2, \quad c_{2}^{(2)}=1 .
\end{gathered}
$$

Here $a$ is a negative parameter.

In this case, conditions (35), which guarantee the existence of a common diagonal functional of the form (19) with identity matrix $P$, are fulfilled with $a<-18 / 29$. Relations (33) and (34) hold if and only if $a<-3 / 5$. Using the results of [15], it is easy to show that if $a<-0.546$, then there exist numbers $\lambda$ and $\mu$ for which the inequalities (30)-(32) hold. 


\section{CONCLUSION}

In this work, we have investigated the conditions for the existence of a common diagonal Lyapunov-Krasovskii functional for a family of linear positive systems with delay. It is well known that the problem of constructing the required functional can be reduced to finding the solution of the corresponding system of linear matrix inequalities. However, such an approach is not always convenient, especially for systems that contain parametric uncertainties. The conditions established in this work are formulated in terms of the feasibility of systems of linear algebraic inequalities, and proofs of the theorems contain constructive algorithms for obtaining the desired functionals. We have shown that our results can be used to analyze the stability of not only linear systems but also some classes of nonlinear differential and difference systems with delay.

As a possible direction for further research, we note an application of the developed approaches to control problems for multi-agent systems with delay and switching communication topology.

\section{ACKNOWLEDGMENTS}

The work was supported by the Governmental Support of Leading Universities of the Russian Federation (grant 08-08), financially supported by the Russian Foundation for Basic Research project no. 16-01-00587-a, Science Foundation Ireland grant 13/RC/2094 and co-funded under the European Regional Development Fund through the Southern \& Eastern Regional Operational Programme to Lero - the Irish Software Research Centre (www.lero.ie).

APPENDIX

Proof of Theorem 1. Let

$$
P=\operatorname{diag}\left\{p_{1}, \ldots, p_{n}\right\}, \quad Q=\operatorname{diag}\left\{q_{1}, \ldots, q_{n}\right\}
$$

where $p_{i}, q_{i}$ are some positive numbers, $i=1, \ldots, n$. Consider the functional (8) corresponding to these matrices. We get

$$
\alpha_{1}\|x(t)\|^{2} \leqslant V\left(x_{t}\right) \leqslant \alpha_{2}\left\|x_{t}\right\|_{\tau}^{2}
$$

Here $\alpha_{1}, \alpha_{2}$ are positive constants, and

$$
\left\|x_{t}\right\|_{\tau}=\sup _{\xi \in[-\tau, 0]}\|x(t+\xi)\|
$$

Fix an index $s \in\{1, \ldots, N\}$, and let us differentiate the functional (8) with respect to the sth subsystem of the family (10). We get

$$
\left.\dot{V}\right|_{(s)}=2 \sum_{i, j=1}^{n} p_{i} a_{i j}^{(s)} x_{i}(t) x_{j}(t)+2 \sum_{i, j=1}^{n} p_{i} b_{i j}^{(s)} x_{i}(t) x_{j}(t-\tau)+\sum_{i=1}^{n} q_{i}\left(x_{i}^{2}(t)-x_{i}^{2}(t-\tau)\right),
$$

where $a_{i j}^{(s)}$ and $b_{i j}^{(s)}$ are elements of the matrices $A_{s}$ and $B_{s}$, respectively.

Suppose now that there exist numbers $\lambda, \mu$ and positive vectors $\theta=\left(\theta_{1}, \ldots, \theta_{n}\right)^{\top}$ and $d=$ $\left(d_{1}, \ldots, d_{n}\right)^{\top}$, that satisfy the conditions of Theorem 1 . Let $p_{i}=d_{i} / \theta_{i}, x_{i}(t)=\theta_{i} z_{i}(t), x_{i}(t-\tau)=$ 
$\theta_{i} y_{i}(t), i=1, \ldots, n$. Then

$$
\begin{gathered}
\left.\dot{V}\right|_{(s)}=2 \sum_{i, j=1}^{n} d_{i} a_{i j}^{(s)} \theta_{j} z_{i}(t) z_{j}(t)+2 \sum_{i, j=1}^{n} d_{i} b_{i j}^{(s)} \theta_{j} z_{i}(t) y_{j}(t)+\sum_{i=1}^{n} \theta_{i}^{2} q_{i}\left(z_{i}^{2}(t)-y_{i}^{2}(t)\right) \\
\leqslant \sum_{i, j=1}^{n} d_{i} a_{i j}^{(s)} \theta_{j}\left(z_{i}^{2}(t)+z_{j}^{2}(t)\right)+\sum_{i, j=1}^{n} d_{i} b_{i j}^{(s)} \theta_{j}\left(z_{i}^{2}(t)+y_{j}^{2}(t)\right)+\sum_{i=1}^{n} \theta_{i}^{2} q_{i}\left(z_{i}^{2}(t)-y_{i}^{2}(t)\right) \\
=\sum_{i=1}^{n} z_{i}^{2}(t)\left(d_{i} \sum_{j=1}^{n}\left(a_{i j}^{(s)}+b_{i j}^{(s)}\right) \theta_{j}+\theta_{i}^{2} q_{i}+\theta_{i} \sum_{j=1}^{n} a_{j i}^{(s)} d_{j}\right)+\sum_{i=1}^{n} \theta_{i} y_{i}^{2}(t)\left(\sum_{j=1}^{n} b_{j i}^{(s)} d_{j}-\theta_{i} q_{i}\right) \\
\leqslant \sum_{i=1}^{n} \theta_{i} z_{i}^{2}(t)\left(\lambda d_{i}+\theta_{i} q_{i}+\sum_{j=1}^{n} a_{j i}^{(s)} d_{j}\right)+\sum_{i=1}^{n} \theta_{i} y_{i}^{2}(t)\left(\sum_{j=1}^{n} b_{j i}^{(s)} d_{j}-\theta_{i} q_{i}\right) .
\end{gathered}
$$

Now let

$$
q_{i}=\frac{1}{\theta_{i}}\left(\max _{s=1, \ldots, N} \sum_{j=1}^{n} b_{j i}^{(s)} d_{j}+\varepsilon\right), \quad i=1, \ldots, n
$$

where $\varepsilon$ is a positive parameter. We get

$$
\left.\dot{V}\right|_{(s)} \leqslant \sum_{i=1}^{n} \theta_{i} z_{i}^{2}(t)\left((\lambda+\mu) d_{i}+\varepsilon\right)-\varepsilon \sum_{i=1}^{n} \theta_{i} y_{i}^{2}(t) .
$$

With sufficiently small values of $\varepsilon$, we have

$$
\left.\dot{V}\right|_{(s)} \leqslant-\alpha_{3} \sum_{i=1}^{n} \theta_{i}^{2} z_{i}^{2}(t)=-\alpha_{3}\|x(t)\|^{2}, \quad \alpha_{3}=\text { const }>0 .
$$

Thus, the constructed functional is a common Lyapunov-Krasovskii functional for the family of subsystems (10), satisfying the requirements of the Krasovskii theorem on asymptotic stability [19] for all $\tau>0$. This completes the proof of Theorem 1 .

Proof of Corollary 2. Let $\gamma=(1, \ldots, 1)^{\top}$. It is easy to show (see $\left.[5,13]\right)$ that if matrices $A_{s}+B_{r}$, $s, r=1, \ldots, N$ are Hurwitz commutative matrices, then the vectors

$$
\theta=(-1)^{N} \prod_{s=1}^{N}\left(A_{s}+B_{s}\right)^{-1} \gamma, \quad d=(-1)^{N} \prod_{s, r=1}^{N}\left(A_{s}^{\top}+B_{r}^{\top}\right)^{-1} \gamma
$$

are positive solutions of systems (12) and (13) respectively.

Applying Corollary 1, we obtain that for the family (10) there exists a common LyapunovKrasovskii diagonal functional of the form (8).

Proof of Theorem 3. Necessity. From the existence of the required functional, it follows that there exists a diagonal positive definite matrix $Q$ such that the quadratic form

$$
W(y, z)=\left(\begin{array}{l}
y \\
z
\end{array}\right)^{\top}\left(\begin{array}{cc}
A^{\top}+A+Q & B \\
B^{\top} & -Q
\end{array}\right)\left(\begin{array}{l}
y \\
z
\end{array}\right)
$$

is negative definite. With $y=z$ we have $W(y, y)=y^{\top}\left(A+B+A^{\top}+B^{\top}\right) y$. This means that matrix (15) must be negative definite. 
Sufficiency. The matrix (15) is Metzler. If it is negative definite, then, according to Statement 1, there exists a positive vector $\zeta$ such that $C \zeta<0$.

We find the diagonal positive definite matrix $Q$ satisfying condition $Q \zeta=B^{\top} \zeta+\varepsilon \gamma$. Here $\varepsilon=$ const $>0, \gamma=(1, \ldots, 1)^{\top}$.

Let

$$
\tilde{L}=\left(\begin{array}{cc}
A^{\top}+A+Q & B \\
B^{\top} & -Q
\end{array}\right)
$$

Then

$$
\tilde{L}\left(\begin{array}{l}
\zeta \\
\zeta
\end{array}\right)=\left(\begin{array}{c}
C \zeta+\varepsilon \gamma \\
-\varepsilon \gamma
\end{array}\right)
$$

If the value of the parameter $\varepsilon$ is small enough, then

$$
\tilde{L}\left(\begin{array}{l}
\zeta \\
\zeta
\end{array}\right)<0
$$

The matrix $\tilde{L}$ is Metzler. Therefore, since inequality (A.1) holds it follows that it is Hurwitz. And since $\tilde{L}$ is a symmetric matrix, its negative definiteness follows from the fact that it is Hurwitz. This completes the proof of Theorem 3.

Proof of Theorem 4. Suppose that for a positive vector $\zeta=\left(\zeta_{1}, \ldots, \zeta_{n}\right)^{\top}$ inequalities (16) are satisfied. Then there exists a number $\delta>0$ such that

$$
\left(A_{s}+B_{s}+A_{s}^{\top}+B_{r}^{\top}\right) \zeta<-\delta \gamma, \quad s, r=1, \ldots, N .
$$

Here $\gamma=(1, \ldots, 1)^{\top}$.

Consider the matrices

$$
\tilde{L}_{s}=\left(\begin{array}{cc}
A_{s}^{\top}+A_{s}+Q & B_{s} \\
B_{s}^{\top} & -Q
\end{array}\right), \quad s=1, \ldots, N,
$$

where elements of the positive definite diagonal matrix $Q=\operatorname{diag}\left\{q_{1}, \ldots, q_{n}\right\}$ satisfy conditions

$$
q_{i} \zeta_{i}=\max _{r=1, \ldots, N} \sum_{j=1}^{n} b_{j i}^{(r)} \zeta_{j}+\varepsilon, \quad i=1, \ldots, n,
$$

and $\varepsilon=$ const $>0$. If $0<\varepsilon<\delta$, then

$$
\tilde{L}_{s}\left(\begin{array}{l}
\zeta \\
\zeta
\end{array}\right) \leqslant\left(\begin{array}{c}
-\delta \gamma+\varepsilon \gamma \\
-\varepsilon \gamma
\end{array}\right)<\left(\begin{array}{l}
0 \\
0
\end{array}\right), \quad s=1, \ldots, N
$$

Using the obtained inequalities, it is easy to show (see the proof of Theorem 3) that the matrices given by (A.2) are negative definite. This completes the proof of Theorem 4.

\section{REFERENCES}

1. Blanchini, F., Colaneri, P., and Valcher, M.E., Switched Positive Linear Systems, Foundat. Trends Syst. Control, 2015, vol. 2, no. 2, pp. 101-273.

2. Rantzer, A., Scalable Control of Positive Systems, Eur. J. Control, 2015, vol. 24, pp. 72-80. 
3. Zhang, J., Huang, J., and Zhao, X., Further Results on Stability and Stabilisation of Switched Positive Systems, IET Control Theory Appl., 2015, vol. 9, no. 14, pp. 2132-2139.

4. Valcher, M.E. and Zorzan, I., On the Consensus of Homogeneous Multiagent Systems with Positivity Constraints, IEEE Trans. Autom. Control, 2017, vol. 62, no. 10, pp. 5096-5110.

5. Farina, L. and Rinaldi, S., Positive Linear Systems: Theory and Applications, New York: Wiley, 2000.

6. Berman, A. and Plemmons, R.J., Nonnegative Matrices in the Mathematical Sciences, Philadelphia: SIAM, 1994.

7. Kazkurewicz, E. and Bhaya, A., Matrix Diagonal Stability in Systems and Computation, Boston: Birkhauser, 1999.

8. Shorten, R.N., Wirth, F., and Leith, D., A Positive Systems Model of TCP-Like Congestion Control, IEEE Trans. Networking, 2006, vol. 14, no. 3, pp. 616-629.

9. Metod vektornykh funktsii Lyapunova $v$ teorii ustoichivosti (Method of Vector Lyapunov Functions in Stability Theory), Voronov, A.A. and Matrosov, V.M., Eds., Moscow: Nauka, 1987.

10. Tkhai, V.N., Model with Coupled Subsystems, Autom. Remote Control, 2013, vol. 74, no. 6, pp. 919-931.

11. Aleksandrov, A.Yu., Chen, Y., Platonov, A.V., and Zhang, L., Stability Analysis and Uniform Ultimate Boundedness Control Synthesis for a Class of Nonlinear Switched Difference Systems, J. Differ. Equat. Appl., 2012, vol. 18, no. 9, pp. 1545-1561.

12. Mason, O., Diagonal Riccati Stability and Positive Time-Delay Systems, Syst. Control Lett., 2012, vol. 61 , no. 1, pp. 6-10.

13. Aleksandrov, A.Yu. and Platonov, A.V., On Absolute Stability of One Class of Nonlinear Switched Systems, Autom. Remote Control, 2008, vol. 69, no. 7, pp. 1101-1116.

14. Aleksandrov, A. and Mason, O., Absolute Stability and Lyapunov-Krasovskii Functionals for Switched Nonlinear Systems with Time-Delay, J. Franklin Inst., 2014, vol. 351, pp. 4381-4394.

15. Pastravanu, O.C. and Matcovschi, M.-H., Max-Type Copositive Lyapunov Functions for Switching Positive Linear Systems, Automatica, 2014, vol. 50, no. 12, pp. 3323-3327.

16. Liberzon, D., Switching in Systems and Control, Boston: Birkhauser, 2003.

17. Shorten, R., Wirth, F., Mason, O., Wulf, K., and King, C., Stability Criteria for Switched and Hybrid Systems, SIAM Rev., 2007, vol. 49, no. 4, pp. 545-592.

18. Vassilyev, S.N. and Kosov, A.A., Analysis of Hybrid Systems' Dynamics using the Common Lyapunov Functions and Multiple Homomorphisms, Autom. Remote Control, 2011, vol. 72, no. 6, pp. 1163-1183.

19. Krasovskii, N.N., On Applications of the Second Lyapunov Method for Equations with Time Delay, Prikl. Mat. Mekh., 1956, vol. 20, no. 3, pp. 315-327.

20. Aleksandrov, A. and Mason, O., Diagonal Riccati Stability and Applications, Linear Algebra Appl., 2016, vol. 492, pp. 38-51.

21. Aleksandrov, A. and Mason, O., Diagonal Lyapunov-Krasovskii Functionals for Discrete-Time Positive Systems with Delay, Syst. Control Lett., 2014, vol. 63, no. 1, pp. 63-67.

22. Narendra, K.S. and Balakrishnan, J., A Common Lyapunov Function for Stable LTI Systems with Commuting A-Matrices, IEEE Transact. Autom. Control, 1994, vol. 39, no. 12, pp. 2469-2471.

23. Liberzon, D., Morse, A.S., and Hespanha, J., Stability of Switched Systems: A Lie Algebraic Condition, Syst. Control Lett., 1999, vol. 37, pp. 117-122.

24. Ebihara, Y., Peaucelle, D., and Arzelier, D., LMI Approach to Linear Positive System Analysis and Synthesis, Syst. Control Lett., 2014, vol. 63, pp. 50-56.

This paper was recommended for publication by B.T. Polyak, a member of the Editorial Board 